\title{
Novel Therapeutic Strategies in Development for Myelodysplastic Syndromes
}

\author{
Nahla A M Hamed* \\ Professor of Hematology, Hematology Department, Faculty of Medicine, Alexandria University, Egypt
}

Submission: May 20, 2019; Published: May 28, 2019

*Correspondence Author: Nahla A M Hamed, Professor of Hematology, Hematology Department, Faculty of Medicine, Alexandria University, Egypt

\begin{abstract}
MDS represents a challenge to the hematologists because of the heterogeneous nature of the disease, and the presence of diversity of conditions across the entire spectrum of MDS as well as the vague boundaries between MDS and other related myeloid disorders. The overall outcome of MDS are very heterogeneous with an overall survival ranges from few months to years in some patients.

Abbreviation: MDS: Myelodysplastic Syndromes; NLRP3: S100A9- Mediated NOD-like receptor Protein 3; CK1 $\alpha$; Casein Kinase 1A1; HMA: Hypomethylating Agents; IST: Immunosuppressive Therapy; HR-MDS: Higher-risk Myelodysplastic Syndromes; TD: Transfusion Dependent; RIC: Reduced Intensity Conditioning; ESAs: Erythropoiesis-Stimulating Agents; PD-1: Programmed Cell Death Protein-1.
\end{abstract}

\section{Introduction}

Myelodysplastic syndromes (MDS) are a heterogeneous group of clonal hematopoietic stem cell neoplasms characterized by ineffective hematopoiesis, dysplasia of the myeloid cells, cytopenias [1] and increased risk of progression to acute leukemia [2]. It occurs more frequently in older individuals [3] with a median age at presentation of 70-75 years. The incidence of MDS is estimated at 5-13/100,000/year [4] but rises to $>20 / 100,000 /$ year in the ageing population [4]. Unexplained anemia associated with a high MCV in the very old should trigger the suspicion of underlying MDS [4].

MDS is a complex multistep process comprising severe disturbance within the hematopoietic cell compartment and in the bone marrow microenvironment and the complex interactions between both compartments [5]. Activation of the innate immune signaling (i.e. myeloid derived suppressor cells) and the activation of NLRP3 inflammasome in hematopoietic stem/ progenitor cells play a central role in the biology of MDS [1] by producing an inflammatory lytic form of cell death termed pyroptosis and clonal expansion [6].

Several prognostic scorings systems risk-stratify MDS patients, including the International Prognostic Scoring System (IPSS), the revised IPSS (IPSS-R), the WHO Prognostic Scoring System (WPSS), and the Global MD Anderson Prognostic Scoring System (MDAPSS).

\begin{abstract}
All use mainly 3 categories:
i. cytogenetic analysis,

ii. bone marrow blast percentage (except for WPSS), and

iii. cytopenias (WPSS uses transfusion dependency, the revised [WPSSR] included hemoglobin level instead of transfusion dependency).
\end{abstract}

Age was included as an independent prognostic factor in MDAPSS and IPSS-R-age only. The IPSS and IPSS-R are the most commonly used prognostic models in clinical practice. Application of these models in practice has several shortcomings [7].

Role of Conventional Drugs, Novel Therapies, and Transplant

Current therapeutics for MDS is primarily based on stratification of patients into lower- and higher-risk disease using clinical prognostic scoring systems [8]. In lower risk MDS, therapy aims at improving cytopenia(s), preventing complications like bleeding or severe infections and to decrease transfusion burden. In higher risk MDS, the principal aim of treatment is to modify the natural course of disease limiting disease progression and improving survival rates [9]. 
Allo-HSCT remains the only treatment option for possible cure. Non-intensive and risk adapted treatment ranging from iron chelation to lenalidomide and hypomethylating agents (HMA) are appropriate for the majority of MDS patients [10]. Lenalidomide and the HMA (azacitidine and decitabine) have been FDA approved for use in MDS-related indications [11]. Once an HMA fails via intolerance, resistance or relapse after favorable response, the patient outlook is poor [11]. MDS in patients over 85 years is not actively managed with pharmacological intervention or chemotherapy [4]. Molecular drivers have profound implications in future clinical investigations, prognosis and treatment decisions [1]. Comprehensive molecular characterization has identified novel therapeutic strategies that offer significant promise [1]. Novel agents in development for MDS include luspatercept, rigosertib, immune checkpoint inhibitors and venetoclax [11].

\section{Lower Risk MDS}

a. Observation is appropriate for asymptomatic patients until their cytopenias worsen or become more symptomatic [11].

b. Patients with anemia and a sEPO < $100 \mathrm{U} / \mathrm{L}$ have a greater than $70 \%$ chance of responding to ESA [11].

c. Lenalidomide: an immunomodulatory drug that selectively suppresses $\operatorname{del}(5 \mathrm{q})$ clones through induction of ubiquitination of casein kinase $1 \mathrm{~A} 1$ (CK1 $\alpha)$ resulting in CK1 $\alpha$ degradation. CK1 is encoded by a gene within a commonly deleted region for $\operatorname{del}(5 q)$ MDS [12]. The "targeted" use of lenalidomide in del5q MDS yielded a 50\% or greater reduction in transfusions in $76 \%$ of patients, whereas $67 \%$ achieved transfusion independence lasting for a median duration of $>2.7$ years [8], and $30-40 \%$ achieved cytogenetic remission [11]. P53 mutations do less well compared with del5q patients without TP53. There is a $27 \%$ response rate to lenalidomide in non del5q patients [8]. Lesser response to lenalidomide treatment occurs with a complex karyotype that includes del5q and with excess marrow blasts [11].

d. Anti-T cell immunosuppressive therapy (IST, e.g., antithymocyte globulin, corticosteroids, and cyclosporine or tacrolimus) is reasonable in patients who lacks excess blasts or a complex karyotype and who has either (i) anemia not responding to ESA or lenalidomide or has (ii) another severe cytopenia not responding to growth factors.

e. IST is a particularly attractive consideration if the marrow is hypocellular for age. The presence of a PNH clone or HLA DR15 status did not predict IST response [11].

f. Treatment with HMA or enrollment in clinical trials is recommended in low-risk MDS patients with anemia who lose response to ESA, danazol, or lenalidomide [13].
Promising New Agents in Clinical Trials for Anemia and Transfusion Dependence Include

\section{Luspatercept}

A specific activin receptor fusion proteins that act as a ligand trap to neutralize negative regulators of late-stage erythropoiesis [9]. It results in RBC transfusion independence in $10 \%$ to $50 \%$ of lower risk MDSs resistant to available treatments [14]. Luspatercept has a substantial potential to target anemia of lower-risk MDS with ring sideroblasts and presence of splice factor SF3B1 mutations [15] who are refractory or not eligible to ESA (NCT02631070, MEDALIST trial) [9].

\section{Roxadustat (FG-4592)}

An oral hypoxia inducible factor prolyl hydroxylase inhibitor. It promotes erythropoiesis through increasing endogenous erythropoietin levels and improves iron regulation by modulation of hepcidin levels. Its efficacy and safety for treating anemia in lower-risk MDS patients with low RBC transfusion burden is in phase 3 study (NCT03263091) [9].

\section{Imetelstat}

A telomerase inhibitor that targets cells with short telomere lengths and active telomerase [9]. MDS patients with significantly shorter telomeres compared to healthy controls and higher telomerase activity exhibited significantly inferior survival [9]. Imetelstat has encouraging activity in lower risk, treatment naïve MDS patients that are RBC-TD [16]. Single center has shown activity of imetelstat in MDS with RS phenotype. Phase 2/3 study is ongoing in RBC-TD and ESA-relapsed or refractory lower-risk MDS patients. Preliminary results demonstrated that $38 \%$ of patients achieved RBC transfusion-independence. Its activity was higher in patients without $\operatorname{del}(5 q)$ and without prior exposure to either lenalidomide or HMA [9].

\section{Higher-Risk Myelodysplastic Syndromes (HR-MDS)}

\section{Allogeneic Hematopoietic Stem Cell Transplantation (Allo-HSCT)}

Allo-HSCT remains the only potentially curative option but is accessible to only a small number of fit patients [10] and is fraught with complications such as acute and chronic graft vs host disease and non-relapse mortality [16]. HMA therapy is used as pre-transplant bridging therapy to cytoreduce disease, especially for those patients who will get reduced intensity conditioning (RIC) approaches and those who have more than $10 \%$ marrow blasts. Outcomes are at least as good with HMA as with intensive chemotherapy [11]. Upfront allo-HSCT has shown no benefit for lower-risk MDS patients, regardless of a myeloablative or RIC strategy [16].

\section{Hypomethylating Agents (HMA)}

HMA are the standards of care in HR-MDS not eligible for allo-HSCT [17]. Azacitidine and decitabine are two nucleoside an- 
alogs that are DNA hypomethylating agents [17]. The mechanism of action of HMA is unclear and may result from a combination of conventional cytotoxic DNA hypomethylation (no specific signature) and immune related mechanisms including changes in interferon signaling and presentation of neoantigens as epitopes to the immune system [11]. HMA can decrease clonal burden and may therefore result in improved hematopoiesis [11]. Response to HMAs cannot be predicted [12]. Available molecular genetic assays do not differentiate responders versus non responders [11].

HMAs dampen immune response by up-regulating inhibitory immune-checkpoint molecule expression such as programmed cell death protein-1 (PD-1), PD-L1, and PD-L2 while enhancing antitumor immune response, resulting in HMA resistance [9]. HMA do not eradicate transformed stem cells, so relapse is inevitable [11]. Patients with HR-MDS and those who fail to respond to or to relapse/ progress after HMA treatment have limited therapeutic options and poor prognosis [18]. The median overall survival of HMA refractory MDS with IPSS intermediate2/ high risk MDS was 6 months [19]. There is no approved second-line therapy [11].

\section{Novel Hypomethylating Agents (HMA)}

Three new HMAs are in late phase in clinical trials: guadecitabine (a parenterally administered dinucleotide nucleoside analog), CC486 (an orally bioavailable form of azacitidine) and cedazurine (an orally administered fixed-dose combination of decitabine and a cytidine deaminase inhibitor) [11].

\section{Novel Combinations Strategies with HMAs}

Current developments include combination of HMA with novel drugs targeting epigenetic or immunomodulatory pathways or prevent resistance by using checkpoint inhibitors to enhance immune attack [12].

i. Immune checkpoint inhibitors (ICPIs): PD-L1 expression is upregulated in HR-MDS compared with lower-risk MDS patients and in those who fail HMA therapy [9]. ICPIs including PD-1 inhibitor nivolumab and cytotoxic T lymphocyte associated antigen 4 (CTLA4) inhibitor ipilimumab have demonstrated activity in the treatment of MDS including those who failed prior HMA therapy [20]. Combining inhibition of the PD-L1/PD-1 pathway with azacytidine may improve outcome [18]. ICPIs toxicities result from overactivation of the immune system [20].

ii. BCL2 inhibitor, venetoclax with azacytidine in HRMDS patients after HMA failure is currently recruiting (NCT02966782) [9].

iii. Isocitrate dehydrogenase (IDH) inhibitors: IDH inhibitors target mechanism in epigenetic regulation. Potential synergistic effects with HMA are assumed and are under investigation [12]. iv. Rigosertib, an inhibitor of Ras effector pathways, is in a phase 3 study in patients failing HMA treatment (NCT02562443) and demonstrated some benefit in a subset of patients [9].

\section{HR-MDS Carrying TP53 Mutations}

TP53 mutations are considered a universally poor prognostic factor. TP53 Mutations have been reported in 5\% to 18\% of MDS patients and are generally associated with higher-risk disease, including MDS with excess blasts, therapy-related myeloid neoplasms, complex cytogenetics and a small subset of patients with $5 \mathrm{q}$ minus syndrome [8]. Patients with HR-MDS carrying TP53 mutations may have clinical response and significant mutation clearance with decitabine treatment [9]. p53 activators restore the wild-type conformation of mutant p53 and thereby rescue $\mathrm{p} 53$ function. APR-246 is a p53 reactivator in phase $1 \mathrm{~b} / 2$ study in combination with azacitidine (NCT03072043) for MDS patients with up to $30 \%$ blasts. Given the poor outcomes in TP53 patient groups, this could be a promising therapeutic agent if the trial yields favorable results [8].

\section{Potential Limitations to Targeted Therapies In MDS}

Polyclonality at diagnosis and clonal evolution through the disease timeline may be the biologic issues that limit targeting single mutations in MDS [8].

\section{Conclusion}

New treatments such as hypomethylating agents and lenalidomide have been approved with a limited benefit to patients' outcome. There is a demand for a complex and personalized variety of therapeutic approaches.

\section{References}

1. Sallman DA, Tanaka TN, List A, Bejar R (2017) SOHO state of the art update and next questions: biology and treatment of myelodysplastic syndrome. Clin Lymphoma Myeloma leuk 17(10):613-620.

2. Selene II, Jose JA, Sardar M, Shah Z, Shafqat M, et al. ( 2018) Histone deacetylase inhibitors in myelodysplastic syndrome. Blood 132: 5528.

3. Lowenberg B (2019) Introduction to a review series on myelodysplastic syndromes. Blood 133(10):1001-1002.

4. McDonald L, McCathy PM, Khan M, Hogan P, Kelleher EC, et al. (2018) Should myelodysplastic syndromes in very old patients be more actively managed? Clinical characteristics, management and outcomes for patients 85 years and older. Blood 132:5515.

5. Banus-Mulet A, Etxabe A, Cornet-Masana JM, Torrente MA, LaraCastillo MC, et al. (2018) Serotonin receptor type 1B constitutes a therapeutic target for MDS and CMML. Scientific Reports 8: 13883.

6. Sallman DA, List A (2019) The central role of inflammatory signaling in the pathogenesis of myelodysplastic syndromes. Blood 133: 10391048.

7. Nazha A (2018) The MDS genomics-prognosis symbiosis. Hematology Am Soc Hematol educ program 2018(1): 270-276.

8. DeZern AE (2018) Treatments targeting MDS genetics: a fool's errand?. Hematology Am Soc Hematol educ program 2018(1): 277-285.

9. Kubasch AS, Platzbecker U (2018) Facing the challenge: Novel treatment options for patients with myelodysplastic syndromes. Educational Updates in Hematology Book 2(S2): 135-137. 


\section{Cancer Therapy \& Oncology International Journal}

10. Platzbecker U (2019) Treatment of MDS. Blood 133: 1096-1107.

11. Steensma DP (2018) Myelodysplastic syndromes current treatment algorithm 2018. Blood Cancer Journal 8: 47.

12. Kubasch AS, Platzbecker U (2018) Beyond the edge of hypomethylating agents: novel combination strategies for older adults with advanced MDS and AML. Cancer 10(6).

13. Gangat N, Patnaik MM, Tefferi A (2016) Myelodysplastic syndromes: Contemporary review and how we treat. Am J Hematol 91(1): 76-89.

14. Fenaux P, Kiladjian JJ, Platzbecker U (2019) Luspatercept for the treatment of anemia in myelodysplastic syndromes and primary myelofibrosis. Blood 133: 790-794.

15. Lindberg EH (2018) Myelodysplastic syndromes. Educational Updates in Hematology Book 2(S2): 127.

This work is licensed under Creative

Commons Attribution 4.0 License

DOI: 10.19080/CTOIJ.2019.13.555874
16. Patnaik MM, Tefferi A (2019) Refractory anemia with ring sideroblasts (RARS) and RARS with thrombocytosis: 2019 update on diagnosis, risk-stratification, and management. Am J Hematol 94(4): 475-488.

17. Duchmann M, Itzykson R (2019) Clinical update on hypomethylating agents. Int J Hematol. https://doi.org/10.1007/s12185-019-02651-9.

18. Gerds AT, Scott BL, Greenberg PL, Khaled SK, Lin TL, et al. (2018) PD-L1 blockade with atezolizumab in higher-risk myelodysplastic syndrome: an initial safety and efficacy analysis. Blood 132: 466.

19. Taylor J, Coleman M, Alvarez K, Pichardo J, Sen F, et al. (2018) Elinexor, a first-in-class XP01 inhibitor, is efficacious and tolerable in patients with myelodysplastic syndromes refractory to hypomethylating agents. Blood 132:233.

20. Rausch CR, Paul S, Bravo GM, Jabbour EJ, Daver NG, et al. (2018) Pattern of immune-mediated toxicities in patients with myelodysplastic syndrome treated with nivolumab and ipilimub. Blood 132: 4367.

\section{Your next submission with Juniper Publishers will reach you the below assets}

- Quality Editorial service

- Swift Peer Review

- Reprints availability

- E-prints Service

- Manuscript Podcast for convenient understanding

- Global attainment for your research

- Manuscript accessibility in different formats

( Pdf, E-pub, Full Text, Audio)

- Unceasing customer service

Track the below URL for one-step submission https://juniperpublishers.com/online-submission.php 